\title{
A model to guide the management and decision of re-planning during radiotherapy for cervical cancer
}

\author{
Wei Zhang ${ }^{1}$, Xiuhua $\mathrm{Li}^{2}$, Tingting $\mathrm{Lin}^{3}$, Fang $\mathrm{Ma}^{4}$, Xiaoyu $\mathrm{Ma}^{4}$, Xiaoli $\mathrm{Wu}^{4}$, Yingming Sun ${ }^{3}$, Xiaoge Sun \\ ${ }^{1}$ Department of Radiation Oncology, Affiliated People's Hospital of Inner Mongolia Medical University, Hohhot, China; ${ }^{2}$ Department of \\ Gynaecology, Fujian Cancer Hospital, The Affiliated Hospital of Fujian Medical University, Fuzhou, China; ${ }^{3}$ Department of Radiation and Medical \\ Oncology, Affiliated Sanming First Hospital of Fujian Medical University, Sanming, China; ${ }^{4}$ Department of Radiation Oncology, Affiliated Hospital \\ of Inner Mongolia Medical University, Hohhot, China \\ Contributions: (I) Conception and design: W Zhang, X Sun, Y Sun; (II) Administrative support: X Wu; (III) Provision of study materials or patients: F \\ Ma, X Ma; (IV) Collection and assembly of data: T Lin, X Li; (V) Data analysis and interpretation: W Zhang; (VI) Manuscript writing: All authors; \\ (VII) Final approval of manuscript: All authors. \\ Correspondence to: Xiaoge Sun. Department of Radiation Oncology, Affiliated Hospital of Inner Mongolia Medical University, Hohhot 010000, China. \\ Email: sunxiaoge789@126.com.
}

\begin{abstract}
Background: To establish a model to predict whether re-planning is needed in the process of cervical cancer radiotherapy.

Methods: We collected the clinical indexes of 132 patients diagnosed with cervical cancer receiving concurrent chemotherapy and radiotherapy, including 33 factors about tumor markers [carcinoembryonic antigen (CEA), cancer antigen 125 (CA-125), squamous cell carcinoma antigen (SCC)], tumor volume, rectal volume, bladder volume, volumes receiving 30-50 Gy in organs-at-risk (OARs), and the maximum dose (Dmax) received by 1-2 cc in OARs. We established a multivariate model for re-planning evaluation via principal component analysis, and then verified the model based on the internal data.

Results: We identified the dose index (P1), tumor size index (P2), and volumes receiving 30-50 Gy in OARs and the tumor $(\mathrm{P} 3)$ as the three most weighted factors of the re-planning model. We set the cut-off for the re-planning modification requirement at 1 . The model was consistent with $\mathrm{R}=0.12 \mathrm{P} 1+0.21 \mathrm{P} 2+0.31 \mathrm{P} 3$, and it performed accurately that area under the test set characteristics curve (AUC) $=0.826]$.
\end{abstract}

Conclusions: Our proposed method can help to reduce image re-examination during treatment, decrease toxicities in OARs, shorten the radiotherapy course, lessen oncologists' efforts, and save medical resources.

Keywords: Cervical cancer; radiotherapy; re-planning; model

Submitted Oct 20, 2021. Accepted for publication Dec 17, 2021.

doi: $10.21037 /$ tcr-21-2545

View this article at: https://dx.doi.org/10.21037/tcr-21-2545

\section{Introduction}

Cervical cancer is one of the most common malignant tumors in women worldwide (1). Radiotherapy is a major treatment for cervical cancer (2). The protection of organsat-risk (OARs) during radiotherapy is essential to prevent side effects (3). Intensity-modulated radiotherapy (IMRT) has superior dose distributions that conform to the shape of the target volume, and most of all, yield lower acute and late radiation toxicities (4). Nevertheless, a study has confirmed that after using IMRT, the radiation toxicities of normal tissue are reduced, but the effect is limited, and the problem cannot be solved completely. For example, Cheng et al. (5) reviewed 222 patients who were treated with IMRT. They demonstrated that the most common toxicity was vaginal stricture (grade 2, n=59, 26.6\%; grade 3, $\mathrm{n}=4,1.80 \%$ ), followed by proctitis (grade 2, $\mathrm{n}=24 ; 10.8 \%$; grade $3, \mathrm{n}=7$; $3.20 \%$ ) and cystitis (grade 2, n=5, 2.3\%; grade 3, n=2; $0.90 \%$ ), and only 5 patients (grade $2,2.3 \%$ ) were found to have colitis. 
The question remains as to the why a toxic reaction of OARs still occurs in IMRT treatment. It has been previously considered that the change in tumor size affects the toxicity of OARs. For example, Chen et al. (6) studied cone-beam computed tomography (CBCT) to measure the tumor changes in 16 patients with cervical cancer, including 23.05 (8.8-46.7), 40.35 (10.3-57.0), 55.57 (25.2-76.6), $64.06(46.9-82.0)$ and $70.85(54.2-87.4) \mathrm{cm}^{3}$ at $9 \mathrm{~Gy} / 5 \mathrm{f}$, 18 Gy/10 f, 27 Gy/15 f, 36 Gy/20 f, and 48.6 Gy/27 f, respectively. The results showed that tumor regression was most obvious at $27 \mathrm{~Gy} / 15 \mathrm{f}$ irradiation. For $36 \mathrm{~Gy} / 20 \mathrm{f}$, tumor regression was not obvious after 20 rounds of radiotherapy. Nam et al. (7) used magnetic resonance imaging to measure the regression of tumor volume in 81 patients with cervical cancer during radiotherapy. The results showed that the tumor regression at 36-45 Gy determined the tumor control rate.

To further reduce the increased toxicity caused by tumor changes, our hospital used re-planning in radiation. However, several reports are inconsistent, suggesting that not all patients need a second plan. Therefore, it is necessary to establish a reliable model to predict whether re-planning is required. Meanwhile, the re-planning prolongs the course of treatment and potentially affects the prognosis. Herein, we pooled the clinical-pathological characteristics and physical parameters of radiotherapy into a principal component analysis and established a mathematical model to guide clinical decisions. We present the following article in accordance with the MDAR reporting checklist (available at https://dx.doi.org/10.21037/tcr-21-2545).

\section{Methods}

\section{Patients}

In this study, 132 patients diagnosed with cervical cancer according to the International Federation of Gynecology and Obstetrics (FIGO) guidelines in 2009 (8) and received image-guided radiotherapy (IGRT) with 4-6 cycles of platinum chemotherapy weekly between May 2017 and December 2019 were included.

The inclusion criteria were as follows: (I) female, 30-80 years old; (II) cervical squamous cell carcinoma was confirmed histologically or pathologically; (III) Karnofsky performance scale $>70$; (IV) expected survival $>3$ months; (V) radiotherapy 29.12 Gy/17 f to 36 Gy/19 f; (VI) patients treated with platinum-based chemotherapy lasting $4-6$ cycles; and (VII) the white blood cells $>3.5 \times 10^{9} / \mathrm{L}$, neutrophil $>1.5 \times 10^{9} / \mathrm{L}$, platelet $>100 \times 10^{9} / \mathrm{L}$, hemoglobin $>90 \mathrm{~g} / \mathrm{L}$, total bilirubin $<1.5$ times the upper limit of the normal value, alanine aminotransferase and aspartate aminotransferase $<2.5$ times the upper limit of the normal value, and creatinine $<1.5$ times the upper limit of normal value.

The exclusion criteria were as follows: (I) patients with mental illness and those unable to cooperate; (II) patients with severe heart, liver, kidney, lung, and other visceral diseases; (III) patients with blood system diseases; (IV) FIGO stage: patients without radiotherapy pointer; (V) patients with other malignant tumors; (VI) patients with leukocytosis caused by infection, immunity, and other diseases. The endpoint of the study was defined as when OARs enter the clinical target volume (CTV), the target area needed to be modified. The study was conducted in accordance with the Declaration of Helsinki (as revised in 2013). The study was performed in the Affiliated Hospital of Inner Mongolia Medical University and approved by the institutional ethics board of Affiliated Hospital of Inner Mongolia Medical University [No. WZ(2021050)] and individual consent for this retrospective analysis was waived.

\section{Radiation procedures}

\section{Positioning scheme}

Patients received oral administration of the compound meglumine diatrizoate before positioning, kept bladder filling and rectal emptiness, were in the supine position, had their hands in front of their foreheads, maintained relaxation of the body, had their legs straightening naturally, thermoplastic film fixed position, and laser lamp calibrating phantom three markers. Next, the cases underwent enhanced CT scanning (scanning thickness: $5 \mathrm{~mm}$; scanning range: from the diaphragm to middle femur). The last CT images were transferred to the treatment planning department.

\section{Target delineation}

Gross target volume (GTV) was defined as a cervical mass that invaded the uterine body and vagina. Lymph node metastasis (GTV-nd) was defined as para-aortic, iliac vascular, pelvic, and inguinal lymph node metastasis. CTV was defined as the drainage area of the uterus, cervix, vagina, and lymph nodes. Planning target volume (PTV) was defined as the expansion of CTV in anterior/posterior, superior/inferior, right/left direction by 7,10 , and $7 \mathrm{~mm}$ (9). 
Table 1 Variable interpretation

\begin{tabular}{|c|c|}
\hline Abbreviations & Full name \\
\hline C (CEA) & Carcinoembryonic antigen \\
\hline CA (CA-125) & Cancer antigen 125 \\
\hline $\mathrm{S}(\mathrm{SCC})$ & Squamous cell carcinoma antigen \\
\hline L1 (long diameter 1) & Long diameter of pre-radiotherapy \\
\hline L2 (long diameter 2) & Long diameter of peri-radiotherapy \\
\hline S1 (short diameter 1) & Short diameter of pre-radiotherapy \\
\hline S2 (short diameter 1) & Short diameter of peri-radiotherapy \\
\hline T1 (tumor volume 1) & Tumor volume of pre-radiotherapy \\
\hline T2 (tumor volume 2) & Tumor volume of peri-radiotherapy \\
\hline R1 (rectal volume 1) & Rectal volume of pre-radiotherapy \\
\hline R2 (rectal volume 2) & Rectal volume of peri-radiotherapy \\
\hline B1 (bladder volume 1) & Bladder volume of pre-radiotherapy \\
\hline B2 (bladder volume 2) & Bladder volume of peri-radiotherapy \\
\hline ED & External dose \\
\hline PX (PTV Dmax) & Maximum planned target dose \\
\hline $\mathrm{RX}$ (rectal Dmax) & Maximum rectal dose \\
\hline BX (bladder Dmax) & Maximum dose of bladder \\
\hline PM (PTV Dmin) & Planned target minimum dose \\
\hline RM (rectal Dmin) & Rectal minimum dose \\
\hline BM (bladder Dmin) & Bladder minimum dose \\
\hline PN (PTV Dmean) & Planned target mean dose \\
\hline RN (rectal Dmean) & Rectal average dose \\
\hline BN (bladder Dmean) & Mean bladder dose \\
\hline R3 (rectal V30) & Volume of rectum $30 \mathrm{~Gy}$ \\
\hline R4 (rectal V40) & Volume of rectum $40 \mathrm{~Gy}$ \\
\hline R5 (rectal V50) & Volume of rectum 50 Gy \\
\hline B3 (bladder V30) & Volume of bladder 30 Gy \\
\hline B4 (bladder V40) & Volume of bladder 40 Gy \\
\hline B5 (bladder V50) & Volume of bladder 50 Gy \\
\hline RC1 (rectal D1cc) & Maximum dose of rectal $1 \mathrm{cc}$ \\
\hline BC1 (bladder D1cc) & Maximum dose of bladder $1 \mathrm{cc}$ \\
\hline RC2 (rectal D2cc) & Maximum dose of rectal $2 \mathrm{cc}$ \\
\hline BC2 (bladder D2cc) & Maximum dose of $2 \mathrm{cc}$ in bladder \\
\hline
\end{tabular}

\section{Restriction of OARs}

Rectum V45 Gy $\leq 50 \%$; bladder V45 Gy $\leq 50 \%$; femoral head V45 Gy $\leq 5 \%$; the Dmax of spinal cord $\leq 45$ Gy; small intestine V25 Gy $\leq 50 \%$, V54 Gy $\leq 2$ cc.

\section{Plan evaluations}

$95 \%$ of PTV met the prescribed dose of the target area. PTV accepted $110 \%$ of the prescription dose (PD)-volume $<20 \%$. PTV accepted $93 \%$ of the PD-volume $<3 \%$. There was no more than $110 \%$ PD anywhere outside the PTV.

\section{Treatment procedures}

After positioning by CT (LightSpeed RT 16, GE, USA), the patient received radiotherapy (1.8-2 Gy/f, 5 fractions per week to 48.5-50 Gy) via a $6 \mathrm{MV}$ high-energy X-ray linear accelerator (Trigoly, Varian, USA). We used CBCT to verify the position and calibrate the settings first, and four cycles of platinum-based chemotherapy were synchronized with radiotherapy once weekly. All procedures performed in this study involving human participants were in accordance with the Declaration of Helsinki (as revised in 2013).

\section{Secondary CT evaluation and re-planning}

CT was used to re-examine all patients after 18 fractions. CT images were matched with the previous plan. If any OAR was included inside the CTV, we re-delineated GTV, CTV, and OARs to adopt the new tumor condition. The dosage of the two-step-plan also accumulated to 48.5-50 Gy.

\section{Statistical analysis}

The R Programming Language x64 3.6.2 (University of Auckland, New Zealand) is selected for all statistical analyses. The original data was normalized to form new data named data, and the relationship between 33 variables (Table 1) in the data and $\mathrm{R}$ (we set $\mathrm{R}$ as the output value to define re-planning situation at a cutoff $=1$ ) was then analyzed. Combined with the principal component analysis (data 1), the main factors affecting the re-planning were identified, and the model was subsequently established. Next, the related problems were explained by polychotomous logistic regression, and finally, the feasibility of the model was verified. 
Table 2 Characteristics of patients

\begin{tabular}{|c|c|}
\hline Characteristics & $\mathrm{N}=132, \mathrm{n}(\%)$ \\
\hline \multicolumn{2}{|l|}{ Age (years) } \\
\hline Range & $31-80$ \\
\hline $30-40$ & $9(6.81)$ \\
\hline $41-50$ & 25 (18.94) \\
\hline $51-60$ & $53(40.15)$ \\
\hline $61-70$ & 37 (28.03) \\
\hline $71-80$ & $8(6.06)$ \\
\hline \multicolumn{2}{|c|}{ Stage (FIGO, 2009) } \\
\hline IB & $5(3.79)$ \\
\hline IIA & $12(9.09)$ \\
\hline IIB & $81(61.36)$ \\
\hline IIIA & $2(1.51)$ \\
\hline IIIB & $29(21.97)$ \\
\hline IVA & $3(2.27)$ \\
\hline \multicolumn{2}{|l|}{ External dose } \\
\hline $46 \mathrm{~Gy} / 25 \mathrm{f}$ & $29(21.97)$ \\
\hline 50 Gy/25 f & $56(42.42)$ \\
\hline $50.96 \mathrm{~Gy} / 28 \mathrm{f}$ & 47 (35.61) \\
\hline \multicolumn{2}{|l|}{ Re-planning } \\
\hline Yes & $24(18.18)$ \\
\hline No & $108(81.82)$ \\
\hline
\end{tabular}

FIGO, International Federation of Gynecology and Obstetrics.

\section{Results}

\section{Characteristics of patients}

One hundred and thirty-two patients with cervical cancer were enrolled in this study, of which 99 were assigned to the training set, and 33 were assigned to the testing set. We collected the patients' characteristics, which are shown in Table 2. The comparison of the patients' tumors, rectums, and bladders pre and peri-radiotherapy is shown in Table 3, and the doses of the rectum, bladder, and tumor are shown in Table 4 .

\section{Some variables were involved in re-planning in radiotherapy}

Polychotomous logistic regression was performed to explore the relationship between 33 variables and replanning (Figure 1). The three criteria show that most of the information of the data set can be preserved by selecting three principal components (Figure 2).

\section{Three indexes were defined to predict the re-planning}

By analyzing the results of the program, we find three main influencing factors. The relationship between them is as shown in Figure 3. We find that: the first index was mainly related to "PN", "PX", "BC1", "BC2", "RX", "RC1", and "RC2", which we defined as the "dose index". The second index was mainly related to "L2", "S2", "S1", "T2", "L1", and "T1", which we defined as the "tumor size index". The

Table 3 Comparison of tumor, rectum, and bladder pre- and peri-radiotherapy

\begin{tabular}{|c|c|c|c|c|c|}
\hline Groups & Tumor volume $\left(\mathrm{cm}^{3}\right)$ & Long diameter (cm) & Short diameter $(\mathrm{cm})$ & Rectal volume $\left(\mathrm{cm}^{3}\right)$ & Bladder volume $\left(\mathrm{cm}^{3}\right)$ \\
\hline Peri-radiotherapy, mean \pm SD & $28.87 \pm 15.02$ & $3.610 \pm 1.207$ & $2.437 \pm 0.823$ & $74.05 \pm 22.71$ & $271.32 \pm 121.5$ \\
\hline
\end{tabular}

The changes of tumor images before and after 17-19 rounds of radiotherapy were compared to evaluate the impact of the above indexes on re-planning.

Table 4 Dose and volume of the rectum, bladder, and PTV

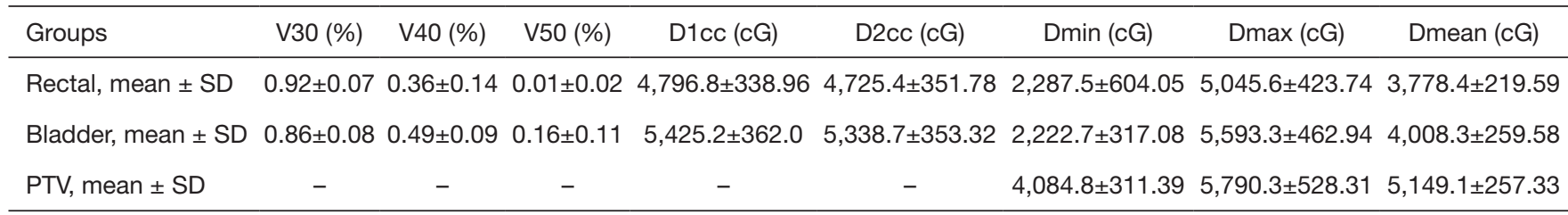

The mean \pm SD of these indexes according to the pre-radiotherapy plan were calculated to evaluate the influence on re-planning. 


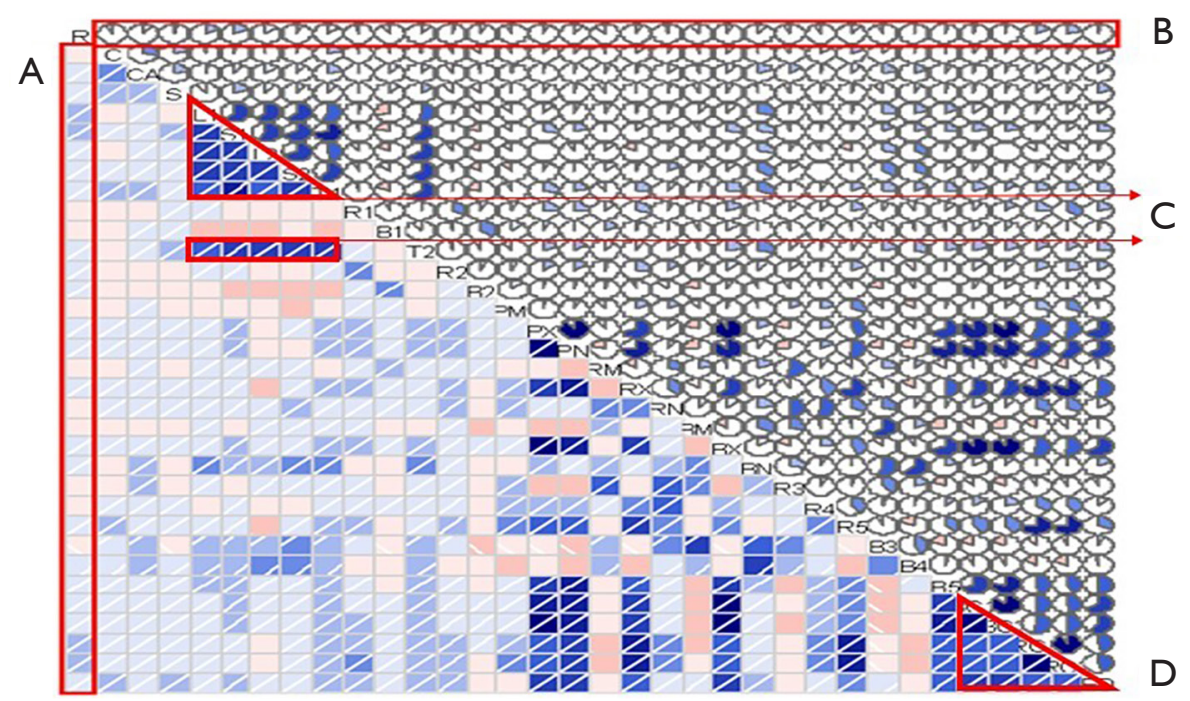

Figure 1 Relationship between 33 variables and re-planning (R). (A) The negative correlation between C, R1, B1, PM, RM, BM, RN, R3, R4, B3, and re-planning was very weak; The positive correlation between L1, S1, RC1, RC2 and re-planning was stronger. (B) Other factors exhibited a weak positive correlation with re-planning. (C) S1, L1, T1, S2, L2, and T2 were positively correlated. (D) BC1, BC2, $\mathrm{RC} 1, \mathrm{RC} 2$, and ED were positively correlated. C, carcinoembryonic antigen; R1, rectal volume of pre-radiotherapy; B1, bladder volume of pre-radiotherapy; PM, planned target minimum dose; RM, rectal minimum dose; BM, bladder minimum dose; RN, rectal average dose; R3, volume of rectum 30 Gy; R4, volume of rectum 40 Gy; B3, volume of bladder 30 Gy; L1, long diameter of pre-radiotherapy; S1, short diameter of pre-radiotherapy; RC1, maximum dose of rectal 1 cc; RC2, maximum dose of rectal 2 cc; S1, short diameter of pre-radiotherapy; L1, long diameter of pre-radiotherapy; T1, tumor volume of pre-radiotherapy; S2, short diameter of peri-radiotherapy; L2, long diameter of peri-radiotherapy; T2, tumor volume of peri-radiotherapy; BC1, maximum dose of bladder $1 \mathrm{cc}$; BC2, maximum dose of 2 cc in bladder; $\mathrm{RC} 1$, maximum dose of rectal $1 \mathrm{cc}$; RC2, maximum dose of rectal $2 \mathrm{cc}$; ED, external dose.

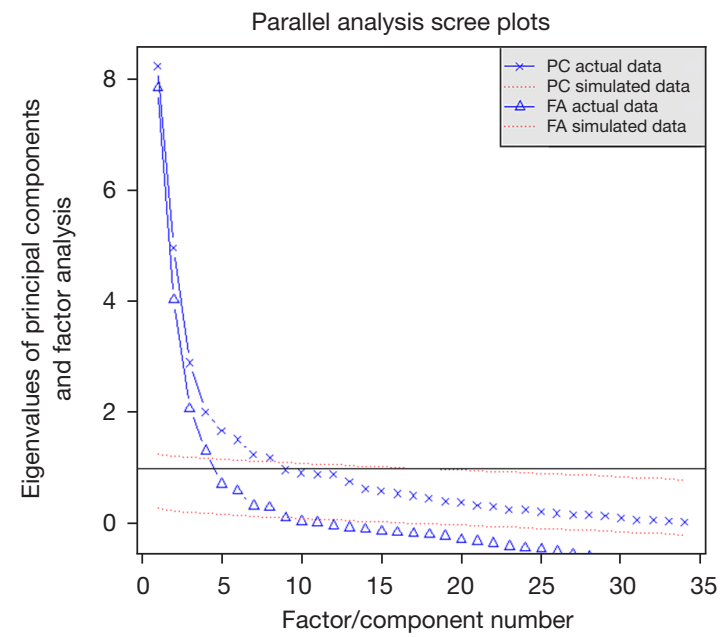

Figure 2 The numbers of main components affecting radiotherapy re-planning. In the gravel test, this paper evaluated three eigenvalue standards based on the average eigenvalue (dotted line) from 200 random data matrices and the eigenvalue standard (horizontal line) greater than 1. PC, principal companents; FA, factors.. third index was mainly related to "R3" and "B3", which we defined as the "V30 index". Their internal relationships are shown in Figures 4-6, respectively.

\section{Establishment of the predictive model for re-planning in radiotherapy}

Given that the system of re-planning contained three indicators (dose index, tumor size index, V30 index), a multi-objective optimization model was first established as follows:

$$
\begin{aligned}
& \operatorname{Max} R=\sum_{i=1}^{3} \lambda_{i} P_{i}^{j} \\
& \lambda_{i} \geq 0 \\
& \sum_{i=1}^{3} \lambda_{i} P_{i}=1
\end{aligned}
$$

\section{Model 1: re-planning index model}

$\lambda_{i}$ was the weighted coefficient of $P_{i} . P_{i}^{j}$ was the $i^{t h}$ principal 


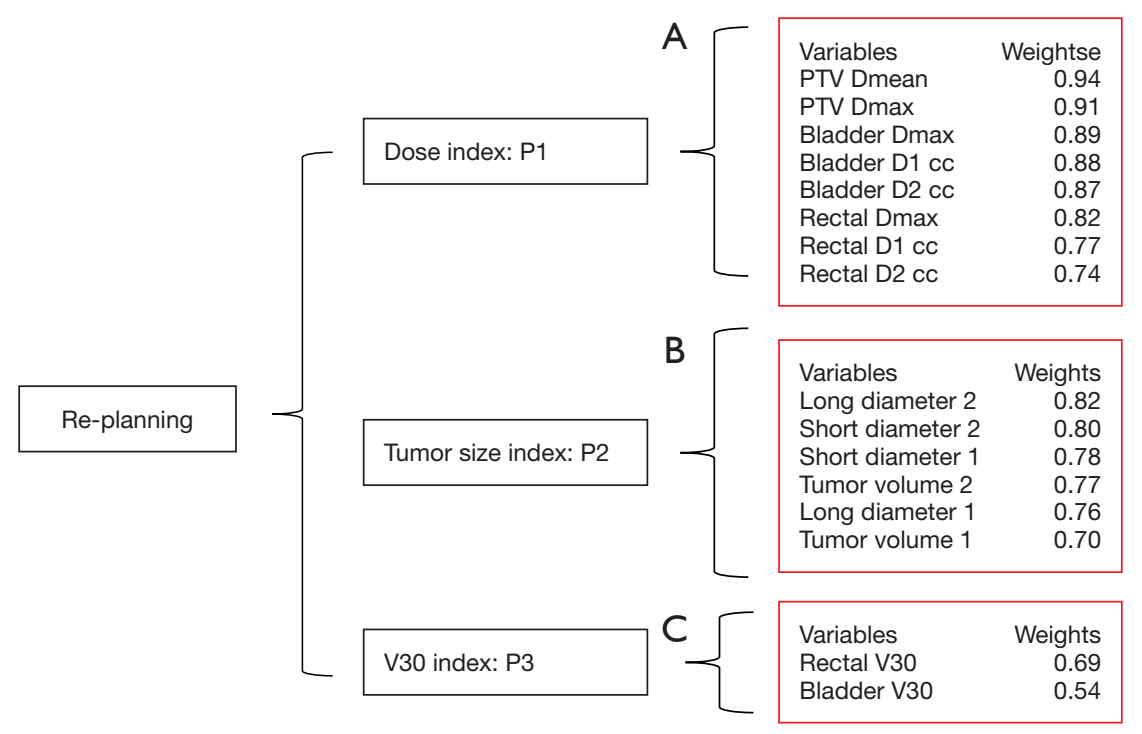

Figure 3 Three principal components were obtained through analysis. (A) In $\mathrm{P}_{1}$, "PTV Dmean" had the greatest influence on re-planning, and other influencing factors were arranged according to weight. (B) In $\mathrm{P}_{2}$, "long diameter 2" had the greatest influence on re-planning, and other influencing factors were arranged according to weight. (C) In $\mathrm{P}_{3}$, "rectal V30" had the greatest influence on re-planning, and other influencing factors were arranged according to weight. PTV, planning target volume; $\mathrm{P}_{1}$, does index; $\mathrm{P}_{2}$, tumor size index; $\mathrm{P}_{3}$, volumes receiving 30 Gy in organs-at-risk (V30).

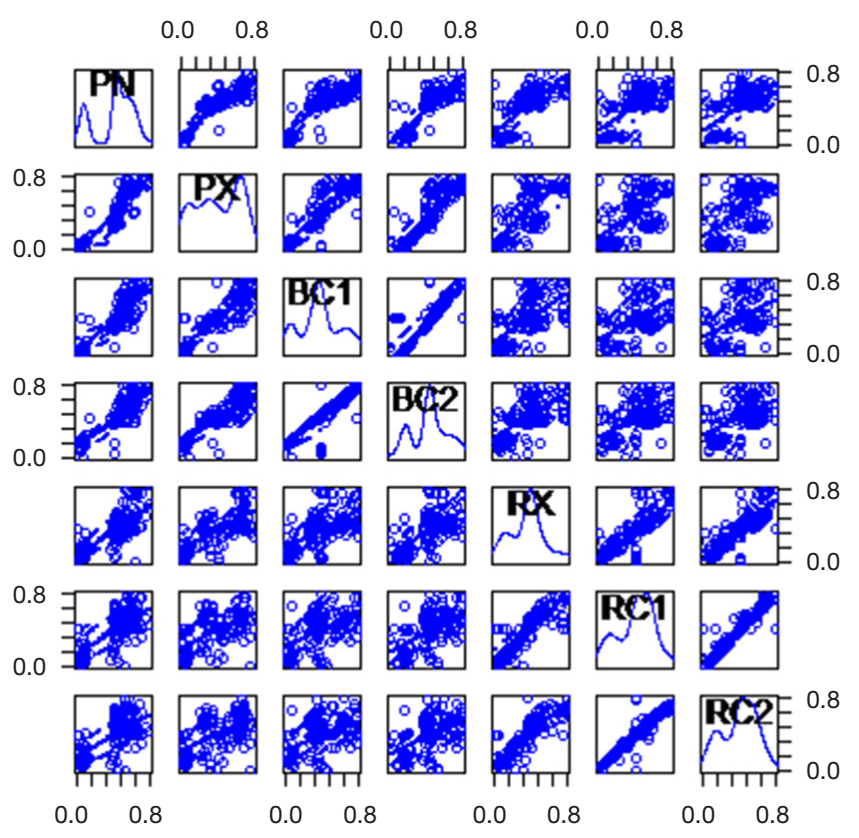

Figure 4 Variable relationships within the "dose index". The diagonal line represents the correlation between the comprehensive analysis of PN, PX, BC1, BC2, RX, RC1, and RC2. The other grids were the result of pairwise comparison based on the diagonal factors. The figure indicated that the factors in the "dose index" were non-linearly correlated. PN, planned target mean dose; PX, maximum planned target dose; $\mathrm{BC} 1$, maximum dose of bladder $1 \mathrm{cc}$; BC2, maximum dose of 2 cc in bladder; RX, maximum rectal dose; RC1, maximum dose of rectal $1 \mathrm{cc}$, RC2, maximum dose of rectal 2 cc. 


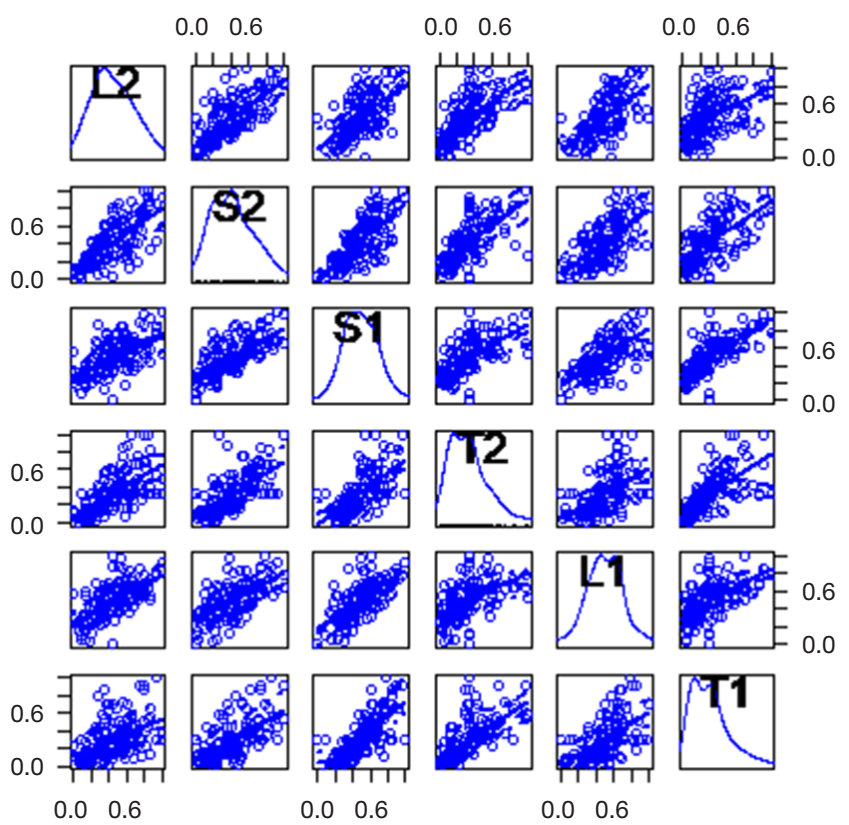

Figure 5 Variable relationships within the "tumor size index". The diagonal line represents the correlation between the comprehensive analysis of L2, S2, S1, T2, L1, and T1. The other grids were the result of pairwise comparison based on the diagonal factors. The figure indicated that the factors in the "tumor size index" were non-linearly correlated. L2, long diameter of peri-radiotherapy; S2, short diameter of peri-radiotherapy; S1, short diameter of pre-radiotherapy; T2, tumor volume of peri-radiotherapy; L1, long diameter of pre-radiotherapy; T1, tumor volume of pre-radiotherapy.
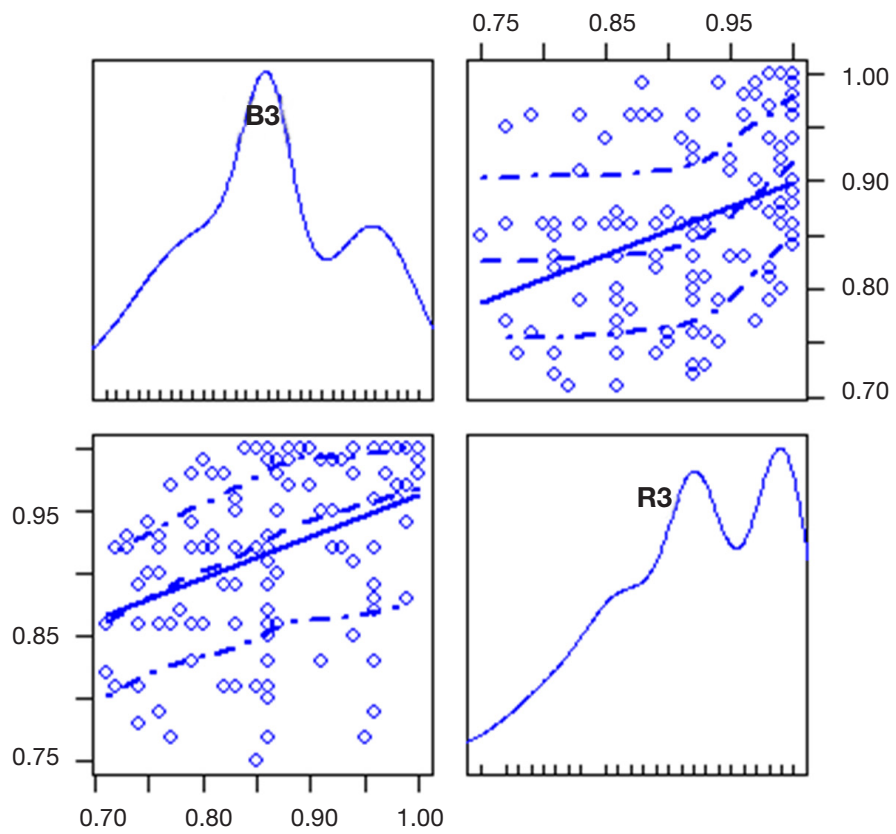

Figure 6 Variable relationships within the "V30 index". The other grids were the result of pairwise comparison based on the diagonal factors. The figure indicated that the factors in the "V30 index" were non-linearly correlated. The diagonal line represents the correlation between the comprehensive analysis of B3 and R3. V30, volumes receiving 30 Gy in organs-at-risk; B3, volume of bladder 30 Gy; R3, volume of rectum $30 \mathrm{~Gy}$. 
Table 5 Top 10 rankings of the three indexes affecting radiotherapy re-planning

\begin{tabular}{lcccc}
\hline Index & $\mathrm{P}_{1}$ & $\mathrm{P}_{2}$ & $\mathrm{P}_{3}$ & $\mathrm{R}$ \\
\hline Rectal Dmax & 0.88 & -0.06 & 0.11 & 0.444 \\
PTV Dmax & 0.87 & 0.18 & -0.23 & 0.443 \\
Bladder Dmax & 0.85 & 0.18 & -0.20 & 0.439 \\
Rectal D2cc & 0.82 & -0.07 & 0.14 & 0.417 \\
Rectal D1cc & 0.83 & -0.07 & 0.09 & 0.412 \\
PTV Dmean & 0.89 & 0.08 & -0.29 & 0.411 \\
Bladder D1cc & 0.81 & 0.16 & -0.23 & 0.407 \\
Bladder D2cc & 0.83 & 0.12 & -0.27 & 0.397 \\
External Dose & 0.68 & 0.20 & -0.05 & 0.390 \\
\hline
\end{tabular}

The three main influencing indexes were obtained, and the effects of the three indexes were comprehensively considered, namely, $\lambda_{1}=0.5 ; \lambda_{2}=0.3 ; \lambda_{3}=0.2$. Among them, $P_{1}, P_{2}$, and $P_{3}$ are the "dose index", "tumor size index", and "V30 index", respectively. PTV, planning target volume.

component index (in this study, we drew the three principal component indexes). $\mathrm{R}$ was the re-planning index. Different patients had different $\lambda_{\mathrm{i}}$. To standardize the data to $\mathrm{P}_{1}$, $\mathrm{P}_{2}$, and $\mathrm{P}_{3}$, we hypothesized that $\mathrm{R}$ was 1 (and that all of the patients underwent re-planning). The final model for predicting re-planning in radiotherapy was as follows: $\mathrm{R}=0.12 \mathrm{P}_{1}+0.21 \mathrm{P}_{2}+0.31 \mathrm{P}_{3}$. The model verified the influence of the three indexes on re-planning and ranked them according to the weights (Table 5). The model also calculated the mostly affected factors in the "dose index" (Table 6), "tumor size index" (Table 7), and "V30 index" (Table 8). At the same time, the $95 \%$ confidence interval (CI) was used to limit each influencing factor (Table 9), which did not differ significantly $(\mathrm{P}>0.05)$.

\section{The predictive ability of the model in the test set}

We utilized the model on the patients in the test set. We randomly selected 33 patients into a test set for $\mathrm{R}$ Programming Language Verification. We calculated the accuracy at 0.826 , indicating that the model performed well in the test set. Thus, this model is applicable to predicting re-planning in radiotherapy.

\section{Discussion}

Cervical cancer is a worldwide pandemic. Early stage
Table 6 Top eight rankings of the dose index affecting re-planning

\begin{tabular}{lcccc}
\hline Index & $\mathrm{P}_{1}$ & $\mathrm{P}_{2}$ & $\mathrm{P}_{3}$ & $\mathrm{R}$ \\
\hline PTV Dmean & 0.89 & 0.08 & -0.29 & 0.89 \\
Rectal Dmax & 0.88 & -0.06 & 0.11 & 0.88 \\
PTV Dmax & 0.87 & 0.18 & -0.23 & 0.87 \\
Bladder Dmax & 0.85 & 0.18 & -0.20 & 0.85 \\
Bladder D2cc & 0.83 & 0.12 & -0.27 & 0.83 \\
Rectal D1cc & 0.83 & -0.07 & 0.09 & 0.83 \\
Rectal D2cc & 0.82 & -0.07 & 0.14 & 0.82 \\
Bladder D1cc & 0.81 & 0.16 & -0.23 & 0.81 \\
\hline
\end{tabular}

Rule out other factors, only consider the effect of dose index on re-planning; that is, $\lambda_{1}=1 ; \lambda_{2}=0 ; \lambda_{3}=0$, in which $\mathrm{P}_{1}, \mathrm{P}_{2}$, and $\mathrm{P}_{3}$ are the "dose index", "tumor size index", and "V30 index", respectively. PTV, planning target volume.

Table 7 Top six rankings of the tumor size index affecting re-planning

\begin{tabular}{lcccc}
\hline Index & $\mathrm{P}_{1}$ & $\mathrm{P}_{2}$ & $\mathrm{P}_{3}$ & $\mathrm{R}$ \\
\hline Short diameter 1 & 0.16 & 0.83 & -0.03 & 0.83 \\
Short diameter 2 & 0.02 & 0.81 & 0.20 & 0.81 \\
Long diameter 2 & -0.11 & 0.80 & 0.09 & 0.80 \\
Tumor volume 1 & 0.16 & 0.77 & 0.09 & 0.77 \\
Long diameter 1 & 0.06 & 0.76 & 0.10 & 0.76 \\
Tumor volume 2 & 0.24 & 0.75 & 0.07 & 0.75 \\
\hline
\end{tabular}

Rule out other factors, only consider the effect of tumor size index on re-planning; that is, $\lambda_{1}=0 ; \lambda_{2}=1 ; \lambda_{3}=0$, in which $\mathrm{P}_{1}, \mathrm{P}_{2}$, and $P_{3}$ are the "dose index", "tumor size index", and "V30 index", respectively.

Table 8 Top six rankings of the V30 index affecting re-planning

\begin{tabular}{lcccc}
\hline Index & $\mathrm{P}_{1}$ & $\mathrm{P}_{2}$ & $\mathrm{P}_{3}$ & $\mathrm{R}$ \\
\hline Rectal V30 & -0.08 & -0.02 & 0.74 & 0.74 \\
Bladder V30 & -0.12 & 0.34 & 0.69 & 0.69 \\
Rectal Dmean & 0.35 & 0.00 & 0.67 & 0.67 \\
Bladder Dmin & -0.18 & 0.09 & 0.57 & 0.57 \\
Rectal Dmin & -0.11 & 0.04 & 0.50 & 0.50 \\
Bladder Dmean & 0.11 & 0.47 & 0.46 & 0.46 \\
Rectal V40 & 0.42 & -0.14 & 0.37 & 0.37 \\
Bladder V40 & -0.15 & 0.43 & 0.34 & 0.34 \\
\hline
\end{tabular}

Rule out other factors, only consider the effect of V30 index on re-planning, that is, $\lambda_{1}=0 ; \lambda_{2}=0 ; \lambda_{3}=1$, in which $\mathrm{P}_{1}, \mathrm{P}_{2}$, and $\mathrm{P}_{3}$ were "dose index", "tumor size index", and "V30 index", respectively. 
Table 9 Ninety-five percent CIs for 16 variables within the three indexes

\begin{tabular}{lccc}
\hline Index & $2.5 \%$ & $97.5 \%$ & $\operatorname{Pr}(>|z|)$ \\
\hline Intercept) & -7.06 & 10.37 & 0.71 \\
Long diameter 1 & -2.10 & 5.33 & 0.38 \\
Short diameter 1 & -2.40 & 6.53 & 0.35 \\
Long diameter 2 & -2.38 & 4.68 & 0.51 \\
Short diameter 2 & -4.63 & 2.96 & 0.65 \\
Tumor volume 1 & -4.77 & 2.73 & 0.65 \\
Tumor volume 2 & -3.08 & 3.84 & 0.85 \\
PTV Dmax & -6.98 & 3.83 & 0.60 \\
PTV Dmean & -3.96 & 6.92 & 0.60 \\
Rectal Dmax & -5.77 & 2.58 & 0.46 \\
Bladder Dmax & -2.38 & 9.53 & 0.27 \\
Bladder D1cc & -8.71 & 2.84 & 0.46 \\
Bladder D2cc & -8.07 & 4.81 & 0.51 \\
Rectal D1cc & -7.13 & 5.19 & 0.85 \\
Rectal D2cc & -1.13 & 11.20 & 0.15 \\
Rectal V30 & -10.61 & 5.63 & 0.54 \\
Bladder V30 & -12.27 & 3.56 & 0.30 \\
\hline
\end{tabular}

The probability on the right side minus on the left gives the $95 \%$ Cls. The coefficient of regression equation is not significant $(\mathrm{P}>0.05)$. $\mathrm{Cl}$, confidence interval; PTV, planning target volume.

patients may be treated by surgical, other patients may be treated by radiotherapy or chemotherapy (10). Radiation treatment is performed using IMRT techniques (volumetric arc, conventional IMRT, or tomotherapy) and threedimensional planned conventional techniques use $6 \mathrm{MV}-\mathrm{X}$ photons (11). Cisplatin $40 \mathrm{mg} / \mathrm{m}^{2}$ body surface area is given in 5 weekly applications to a total dose of $200 \mathrm{mg} / \mathrm{m}^{2}$ during external beam radiation. In case of contraindication to cisplatin, carboplatin (area under curve 2-weekly) is applied (12). Even the radiation technique used was IMRT, genitourinary and gastrointestinal toxicity had hurt patients. In order to reduce these damage, we established a mathematical model to guide secondary planning during radiotherapy for cervical cancer.

In this paper, notably, "dose", "tumor size", and "V30" were found to be the major impacting factors in evaluating the necessity of re-planning. Furthermore, the model performed well in the test set. The goal of radiation oncologists is to deliver a high dose X-ray to foci and minimize the X-ray uptake of normal tissue. Meantime, the radiation-induced injuries of OARs are restricted. So, it is imperative to understand the relative anatomical position between the tumor and paracancerous tissue both pre- and peri-radiotherapy. However, the relative position of OAR tends to move during radiotherapy as tumors shrink.

Therefore, in this study, we typically performed twostage CT during radiotherapy (18 times; 30.6-34 Gy/17 f) to evaluate the necessity of secondary planning of radiotherapy, in order to determine the tumor volume, location changes, and OARs. As previously reported, CBCT is limited and cannot solve the problem completely $(13,14)$. Meanwhile, some studies have concluded that CBCT is an effective means to reduce positioning error and directly evaluate the axial and horizontal position changes of the tumor, bladder, and rectum during treatment (15-17). So, the most important subsidiary condition is that CBCT is applied weekly for every patient. However, Mason et al. (18) used the Dice similarity coefficient and measured contourto-contour distance to evaluate spatial image changes, so as to improve the treatment accuracy of cervical cancer by adaptive radiotherapy. CBCT has a small, lightweight, and open architecture, which can be directly integrated into the linear accelerator (19). However, it is unable to reduce the dosage distribution in OAR $(20,21)$.

CEA, CA-125, and SCC have been verified to be related to the tumor burden and could predict tumor progression $(22,23)$. However, considering our limited sample size, we could not find the expression diversity of CEA, CA-125, and SCC in radiotherapy planning. So, we eliminated clinicalpathological characteristics to build our model.

"Short diameter 1", as the most influencing factor of tumor regression, could be a potential factors affecting re-planning. As previously reported $(24,25)$, the change of tumor volume affects the radioactive side effects to the bladder and rectum, which seems to indicate that the volume change of the bladder and rectum are negatively correlated with the change in tumor volume. This study found that the change in tumor size affected the patient's original radiotherapy plan. Thus, we needed to modify the plan to reduce the damage to OARs.

"PTV Dmean" is a crucial factor in evaluating a radiation plan. Many scholars report that in an optimal plan, for the target area, the Dmin should be close to the PD, so that the PD coverage rate is close to 1 (26). Also, the maximum and average doses for normal tissue should be minimize to ensure that the smaller exposure volume is within the tolerance threshold points (27). Our findings partly confirm 
the above. We demonstrated that the average dose of PTV affects the related dose index of the rectum and has a significant positive correlation. Altogether, the "PTV Dmean" should be reduced in order to protect the rectum.

Besides the tumor size and "PTV Dmean", "V30" was also found to be involved in re-planning (28). Qiao et al. (29) reported that the "V30" can predict injury to OARs, which can be reduced by reducing the "V30". Previous studies have shown that the larger the "V30", the more serious the side effects and the larger the intestinal volume (30-33). Unsurprisingly, "V30" affects enteritis and is significantly positively correlated to rectal volume. Therefore, the "V30" should be reduced in order to protect the rectum.

The model proposed in this paper has similar defects with a mass model, such as the end-to-end OARs segmentation model evaluated by Liu et al. (34), in providing accurate and consistent OARs segmentation results in much less time. The disadvantage of our model is that it is a single-center design, which means that the model would not apply to patients in other hospitals. Even though this model has achieved perfect results, it still required the collection of multiple data sources. However, the advantage of our model is that it can integrate medical knowledge and safely and reasonably evaluate whether the radiotherapy plan needs to be changed. Moreover, it can further reduce the damage of OAR. In short, modern radiotherapy needs to combine big data with medical treatment to deal with the uncertainty implied in radiotherapy and plan treatment more sensibly.

\section{Acknowledgments}

We would like to thank Dr. Fu Zhang from Shanghai Jiao Tong University in China for his technical support with the R Programming Language.

Funding: None.

\section{Footnote}

Reporting Checklist: The authors have completed the MDAR reporting checklist. Available at https://dx.doi. org/10.21037/tcr-21-2545

Data Sharing Statement: Available at https://dx.doi. org/10.21037/tcr-21-2545

Conflicts of Interest: All authors have completed the ICMJE uniform disclosure form (available at https://dx.doi. org/10.21037/tcr-21-2545). The authors have no conflicts of interest to declare.

Ethical Statement: The authors are accountable for all aspects of the work in ensuring that questions related to the accuracy or integrity of any part of the work are appropriately investigated and resolved. The study was conducted in accordance with the Declaration of Helsinki (as revised in 2013). The study was performed in the Affiliated Hospital of Inner Mongolia Medical University and approved by the institutional ethics board of Affiliated Hospital of Inner Mongolia Medical University [No. WZ(2021050)] and individual consent for this retrospective analysis was waived.

Open Access Statement: This is an Open Access article distributed in accordance with the Creative Commons Attribution-NonCommercial-NoDerivs 4.0 International License (CC BY-NC-ND 4.0), which permits the noncommercial replication and distribution of the article with the strict proviso that no changes or edits are made and the original work is properly cited (including links to both the formal publication through the relevant DOI and the license). See: https://creativecommons.org/licenses/by-nc-nd/4.0/.

\section{References}

1. Bray F, Ferlay J, Soerjomataram I, et al. Global cancer statistics 2018: GLOBOCAN estimates of incidence and mortality worldwide for 36 cancers in 185 countries. CA Cancer J Clin 2018;68:394-424.

2. Cibula D, Pötter R, Planchamp F, et al. The European Society of Gynaecological Oncology/European Society for Radiotherapy and Oncology/European Society of Pathology guidelines for the management of patients with cervical cancer. Radiother Oncol 2018;127:404-16.

3. Bandanatham S, Gururajachar JM, Somashekar MK. Compliance with bladder protocol during concurrent chemoradiation for cancer of the cervix and its impact on enteritis: A prospective observational study. Rep Pract Oncol Radiother 2018;23:69-74.

4. Sun Y, Liu G, Chen W, et al. Dosimetric comparisons of craniospinal axis irradiation using helical tomotherapy, volume-modulated arc therapy and intensity-modulated radiotherapy for medulloblastoma. Transl Cancer Res 2019;8:191-202.

5. Cheng JY, Huang EY, Hsu SN, et al. Simultaneous integrated boost (SIB) of the parametrium and cervix in 
radiotherapy for uterine cervical carcinoma: a dosimetric study using a new alternative approach. Br J Radiol 2016;89:20160526.

6. Chen W, Bai P, Pan J, et al. Changes in Tumor Volumes and Spatial Locations Relative to Normal Tissues During Cervical Cancer Radiotherapy Assessed by Cone Beam Computed Tomography. Technol Cancer Res Treat 2017;16:246-52.

7. Nam H, Park W, Huh SJ, et al. The prognostic significance of tumor volume regression during radiotherapy and concurrent chemoradiotherapy for cervical cancer using MRI. Gynecol Oncol 2007;107:320-5.

8. Pecorelli S, Zigliani L, Odicino F. Revised FIGO staging for carcinoma of the cervix. Int J Gynaecol Obstet 2009;105:107-8.

9. Taylor A, Rockall AG, Reznek RH, et al. Mapping pelvic lymph nodes: guidelines for delineation in intensitymodulated radiotherapy. Int J Radiat Oncol Biol Phys 2005;63:1604-12.

10. Zhou H, Li Q, Xu C, et al. Prognosis of stage III cervical cancer: a two-way outcome study. Transl Cancer Res 2020;9:2565-75.

11. Yang J, Cai H, Xiao ZX, et al. Effect of radiotherapy on the survival of cervical cancer patients: An analysis based on SEER database. Medicine (Baltimore) 2019;98:e16421.

12. Marnitz S, Tsunoda AT, Martus P, et al. Surgical versus clinical staging prior to primary chemoradiation in patients with cervical cancer FIGO stages IIB-IVA: oncologic results of a prospective randomized international multicenter (Uterus-11) intergroup study. Int J Gynecol Cancer 2020;30:1855-61.

13. Mullaney LM, O'Shea E, Dunne MT, et al. A randomized trial comparing bladder volume consistency during fractionated prostate radiation therapy. Pract Radiat Oncol 2014;4:e203-12.

14. Gunnlaugsson A, Kjellén E, Nilsson P, et al. Dosevolume relationships between enteritis and irradiated bowel volumes during 5-fluorouracil and oxaliplatin based chemoradiotherapy in locally advanced rectal cancer. Acta Oncol 2007;46:937-44.

15. van de Bunt L, van der Heide UA, Ketelaars M, et al. Conventional, conformal, and intensity-modulated radiation therapy treatment planning of external beam radiotherapy for cervical cancer: The impact of tumor regression. Int J Radiat Oncol Biol Phys 2006;64:189-96.

16. Tyagi N, Lewis JH, Yashar CM, et al. Daily online cone beam computed tomography to assess interfractional motion in patients with intact cervical cancer. Int J Radiat Oncol Biol Phys 2011;80:273-80.

17. Dzierma Y, Beyhs M, Palm J, et al. Set-up errors and planning margins in planar and CBCT image-guided radiotherapy using three different imaging systems: A clinical study for prostate and head-and-neck cancer. Phys Med 2015;31:1055-9.

18. Mason SA, White IM, O'Shea T, et al. Combined Ultrasound and Cone Beam CT Improves Target Segmentation for Image Guided Radiation Therapy in Uterine Cervix Cancer. Int J Radiat Oncol Biol Phys 2019;104:685-93.

19. Rodgers J, Hales R, Whiteside L, et al. Comparison of radiographer interobserver image registration variability using cone beam CT and MR for cervix radiotherapy. Br J Radiol 2020;93:20200169.

20. van de Bunt L, Jürgenliemk-Schulz IM, de Kort GA, et al. Motion and deformation of the target volumes during IMRT for cervical cancer: what margins do we need? Radiother Oncol 2008;88:233-40.

21. Miyasaka Y, Kadoya N, Umezawa R, et al. Comparison of predictive performance for toxicity by accumulative dose of DVH parameter addition and DIR addition for cervical cancer patients. J Radiat Res 2021;62:155-62.

22. Wagner RK, Atzinger A, Breit A. The monitoring of gynecological radiotherapy using serial tumor marker determinations. Strahlenther Onkol 1990;166:446-52.

23. Sarandakou A, Phocas I, Botsis D, et al. Tumour-associated antigens CEA, CA125, SCC and TPS in gynaecological cancer. Eur J Gynaecol Oncol 1998;19:73-7.

24. Amini A, DeWitt PE, Vinogradskiy Y, et al. Should we customize PTV expansions for BMI? Daily cone beam computerized tomography to assess organ motion in postoperative endometrial and cervical cancer patients. Rep Pract Oncol Radiother 2016;21:195-200.

25. Herrera FG, Callaway S, Delikgoz-Soykut E, et al. Retrospective feasibility study of simultaneous integrated boost in cervical cancer using Tomotherapy: the impact of organ motion and tumor regression. Radiat Oncol 2013;8:5.

26. Chaikh A, Balosso J. The use of TCP based EUD to rank and compare lung radiotherapy plans: in-silico study to evaluate the correlation between TCP with physical quality indices. Transl Lung Cancer Res 2017;6:366-72.

27. K Jensen NB, Pötter R, Spampinato S, et al. Dose-Volume Effects and Risk Factors for Late Diarrhea in Cervix Cancer Patients After Radiochemotherapy With Image Guided Adaptive Brachytherapy in the EMBRACE I 
Study. Int J Radiat Oncol Biol Phys 2021;109:688-700.

28. Chopra S, Krishnatry R, Dora T, et al. Predictors of late bowel toxicity using three different methods of contouring in patients undergoing post-operative radiation for cervical cancer. Br J Radiol 2015;88:20150054.

29. Qiao L, Cheng J, Liang N, et al. A comparative dosimetric study of volumetric-modulated arc therapy vs. fixed field intensity-modulated radiotherapy in postoperative irradiation of stage IB-IIA high-risk cervical cancer. Oncol Lett 2016;11:959-64.

30. Murthy V, Master Z, Adurkar P, et al. 'Plan of the day' adaptive radiotherapy for bladder cancer using helical tomotherapy. Radiother Oncol 2011;99:55-60.

31. Masters BJ, Pascoe A, Walker G, et al. Radiobiology of Acute Rectal Toxicity. Clin Oncol (R Coll Radiol)

Cite this article as: Zhang $\mathrm{W}, \mathrm{Li} \mathrm{X}, \mathrm{Lin} \mathrm{T}, \mathrm{Ma} F, \mathrm{Ma} X, \mathrm{Wu} \mathrm{X}$, Sun $\mathrm{Y}$, Sun X. A model to guide the management and decision of re-planning during radiotherapy for cervical cancer. Transl Cancer Res 2021;10(12):5352-5363. doi: 10.21037/tcr-21-2545
2018;30:594-5.

32. Reis T, Khazzaka E, Welzel G, et al. Acute small-bowel toxicity during neoadjuvant combined radiochemotherapy in locally advanced rectal cancer: determination of optimal dose-volume cut-off value predicting grade 2-3 diarrhoea. Radiat Oncol 2015;10:30.

33. Miyanaga N, Akaza H, Okumura T, et al. A bladder preservation regimen using intra-arterial chemotherapy and radiotherapy for invasive bladder cancer: a prospective study. Int J Urol 2000;7:41-8.

34. Liu Z, Liu X, Xiao B, et al. Segmentation of organs-at-risk in cervical cancer $\mathrm{CT}$ images with a convolutional neural network. Phys Med 2020;69:184-91.

(English Language Editor: A. Kassem) 